\title{
Anisotropic absorption and reduced scattering spectra of chicken breast tissue measured using oblique incidence reflectometry
}

Lihong V. Wang, Guillermo Marquez, Sharon L. Thomsen

Lihong V. Wang, Guillermo Marquez, Sharon L. Thomsen, "Anisotropic absorption and reduced scattering spectra of chicken breast tissue measured using oblique incidence reflectometry," Proc. SPIE 3250, Optical Biopsy II, (16 April 1998); doi: 10.1117/12.305386

Event: BiOS '98 International Biomedical Optics Symposium, 1998, San Jose, CA, United States 


\title{
Anisotropic absorption and reduced scattering spectra of chicken breast tissue measured using oblique incidence reflectometry
}

\author{
Lihong V. Wang, Ph.D. ${ }^{a}$ \\ Guillermo Marquez, B. S. \\ Optical Imaging Laboratory, Biomedical Engineering Program \\ Texas A\&M University, College Station, Texas 77843-3120, USA \\ Sharon L. Thomsen, M. D. \\ Laser Biology Research Laboratory \\ University of Texas M. D. Anderson Cancer Center \\ 1515 Holcombe Boulevard, Houston, Texas 77030, USA
}

\begin{abstract}
Oblique incidence reflectometry is a simple and accurate method for measuring the absorption and reduced scattering coefficients of turbid media. We used this technique to deduce absorption and reduced scattering spectra from wavelength-resolved measurements of the relative diffuse reflectance profile of white light as a function of source-detector distance. In this study we measured the absorption and reduced scattering coefficients of chicken breast tissue in the visible range $(400-800 \mathrm{~nm})$ with the oblique incidence probe oriented at 0 and 90 degrees relative to the muscle fibers. We found that the deduced optical properties varied with the probe orientation. This experiment demonstrated the application of oblique-incidence, fiber-optic reflectometry to measurements on biological tissues and the effect of tissue structural anisotropy on optical properties.
\end{abstract}

\section{Key Words}

Oblique incidence, reflectometry, optical properties, turbid media, biological tissues.

(a) Corresponding author. E-mail: LWang@tamu.edu. URL: http://biomed.tamu.edu/ lw. 


\section{Introduction}

This paper presents the continued research for measuring optical properties of turbid media using oblique incidence reflectometry. Wang and Jacques first presented this method as a means of measuring the reduced scattering coefficient of biological tissues at a single wavelength ${ }^{1}$ and Lin et al. have demonstrated that absorption and scattering spectra can be easily obtained with this method as well ${ }^{2}$. Our current work is based on finding the relationship of tissue optical properties to muscle fiber orientation. ${ }^{3,4}$

Measuring the optical properties, absorption coefficient $\left(\mu_{\mathrm{a}}\right)$ and reduced scattering coefficient $\left(\mu_{\mathrm{s}}^{\prime}\right)$, of biological tissues non-invasive, in vivo, and real-time remains a problem in the field of biomedical optics. It is important to know the optical properties of the tissues being irradiated to properly dose light in the therapeutic procedures such as photocoagulation or photodynamic therapy or to measure tissue metabolic status. Also the optical properties themselves can potentially provide enough information to diagnose disease, in particular cancer. This is because light in the visible and near infrared interacts with both the molecular, and subcellular components of tissue. Therefore, characteristic changes in tissue optical properties at particular wavelengths may be indicative of disease.

Other techniques have been developed to measure tissue optical properties. The most common method is the integrating sphere measurement, but this requires the removal of a thin slice of tissue (invasive) ${ }^{5}$. Another technique is normal incidence video reflectometry. One variation of normal incidence reflectometry which is based on diffusion theory provides a non-invasive way to determine optical properties, but requires a measurement of both the spatial distribution of diffuse reflectance and the total diffuse reflectance at each wavelength ${ }^{6}$. In short, noninvasive, inexpensive, and real time measurement of tissue optical properties remains a challenge.

\section{Methods and Materials}

\section{Experimental}

The experimental system is drawn schematically in Figure 1. The detection system was an optical multichannel analyzer that utilizes a spectrograph, an intensified CCD camera, and a computer to automatically scan through the wavelength range of $400 \mathrm{~nm}-800 \mathrm{~nm}$ and give the spectrum of the collected light. 
White light was delivered and the reflectance was collected with a fiber optic probe made from black delrin and $600 \mu \mathrm{m}$ diameter low-loss optical fiber. The source fiber is oriented at $45^{\circ}$ angle, and nine (9) collection fibers in a linear array collected the diffuse reflectance (Figure 2). To correct for slight variations in collection efficiency from one detection fiber to the next, a correction factor, $\mathrm{k}_{\mathrm{i}}$, was previously calculated for each fiber based on a calibration procedure using standard phantoms.

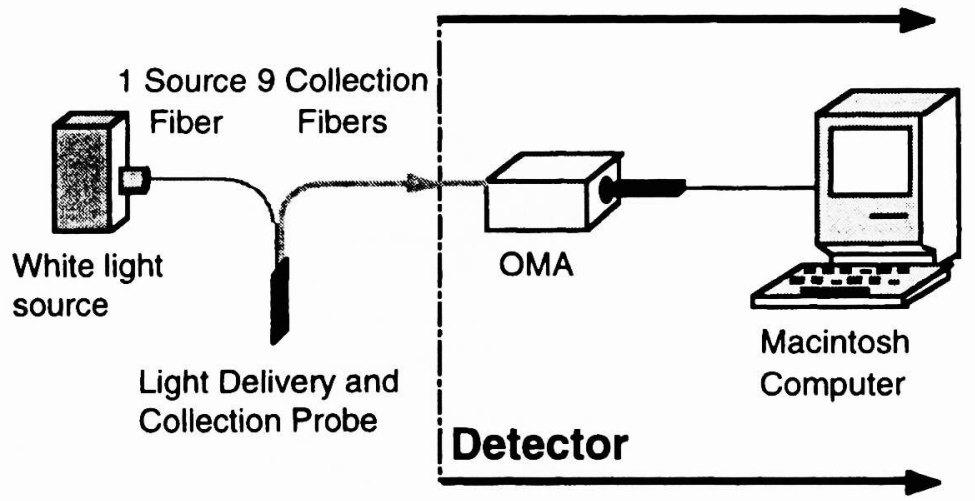

Figure 1. Schematic of experimental apparatus. White light is coupled to the source fiber which delivers light to the chicken breast tissue. The diffuse reflectance is collected by 9 collection fibers and detected by the OMA.
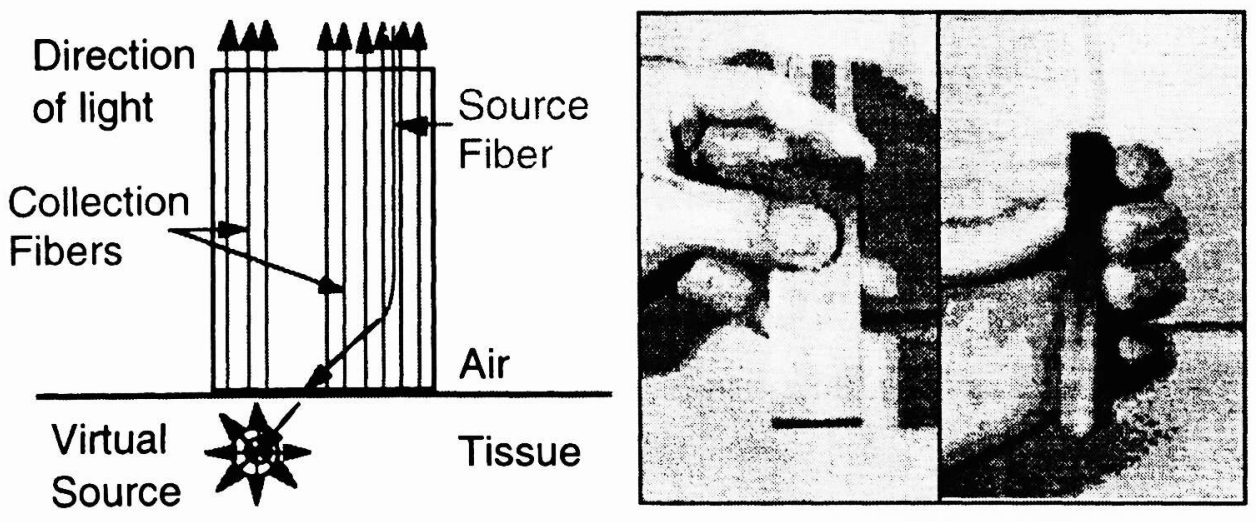

Figure 2. Oblique-incidence optical fiber probe. One source fiber incident at $45^{\circ}$ and nine collection fibers were bundled and encased to form a hand-held piece. The core diameter of the fibers was $600 \mu \mathrm{m}$. The index of refraction of the fiber core was 1.46. The index of refraction of chicken breast tissue was 1.37 . The angle of refraction was computed to be $48.9^{\circ}$. On the right is an actual picture of the probe.

Frozen, raw, chicken breasts from the store were allowed to thaw. The skin and fat was removed and the chicken breast were cut so as to have only one fiber orientation per chicken breast. The pieces were stacked in a small black bucket with all muscle fibers aligned (Figure 3 ). 

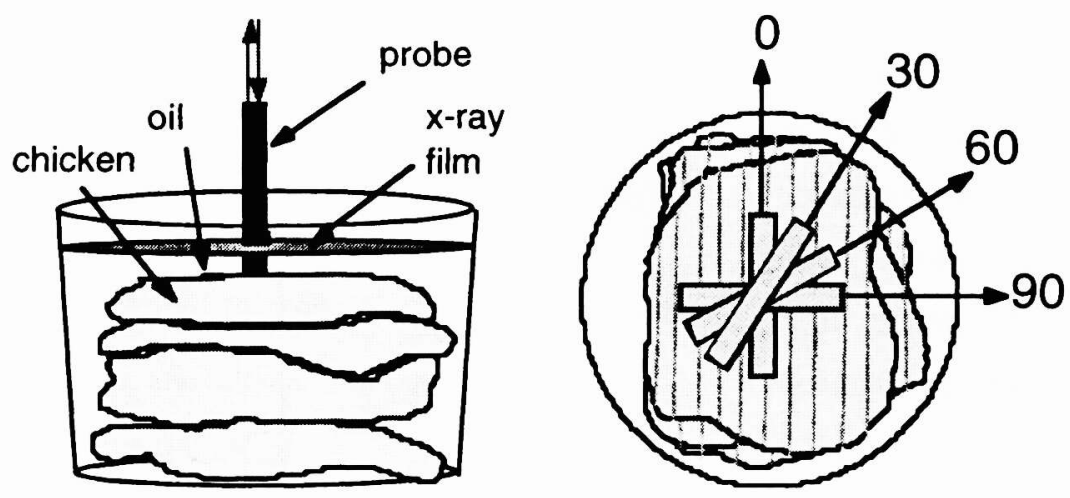

Figure 3. Chicken breast tissue setup. The container is 4" deep and 4" diameter. The probe made physical contact with the chicken tissue. Immersion oil was used as a coupling media. The figure on the right, shows the muscle orientation with respect to the probe: $0^{\circ}$ indicates parallel and $90^{\circ}$ perpendicular.

The bucket was then filled with small amount of microscope immersion oil $(n=1.5105)$ to ensure good coupling between the separate pieces of chicken and to index match to the top surface. On top of the oil we floated an exposed $\mathrm{x}$-ray film to approximate a matched boundary condition for diffusion theory. Two measurements were performed with the probe oriented at different angles relative to the muscle fibers: $0^{\circ}$ (parallel) and $90^{\circ}$ (perpendicular).

\section{Theoretical}

When light enters a semi-infinite tissue, it will generally scatter a number of times before either being absorbed or escaping the tissue surface at a point other than its point of entry. This scattered light that escapes is called diffuse reflectance. Our method is derived from a diffusion theory based model of diffuse reflectance ${ }^{7}$. This diffusion theory model does not accurately model near diffuse reflectance. That is, reflectance that falls within the range of 1-2 transport mean free paths (mfp') of the source. This problem can be avoided by using only the "far diffuse reflectance."

For normally incident light, the spatial distribution of diffuse reflectance has been modeled by two isotropic point sources; one positive source located below the tissue surface and one negative image source above the tissue surface ${ }^{7}$. If light is delivered obliquely, the positive source is buried at the same distance from the point of incidence but with depth determined by Snell's law. Therefore, the fundamental difference between normal and oblique incidence is a shift in the positions of the point sources in the $x$ direction (Figure 4). Wang and Jacques' found the depth of the buried source to be

$$
3 \mathrm{D}=1 /\left(0.35 \mu_{\mathrm{a}}+\mu_{\mathrm{s}}^{\prime}\right)
$$




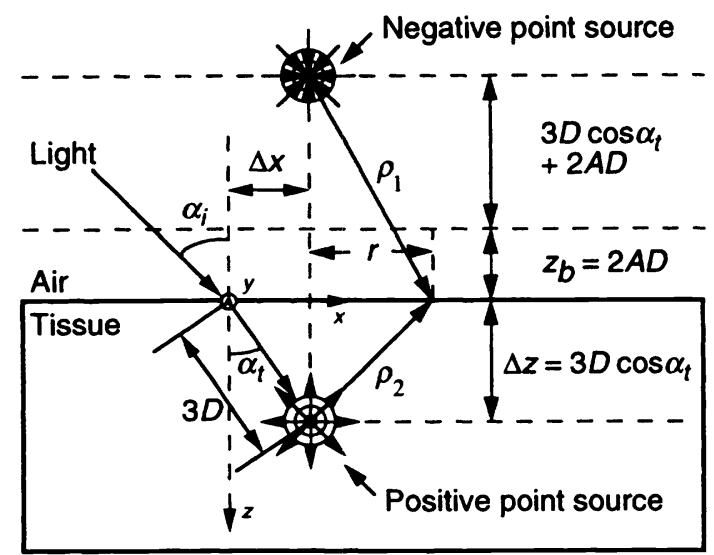

Figure 4. Schematic representation of obliquely incident light.

The height of the image source is further determined by the boundary condition at the tissue surface. The modified dipole source diffusion theory model is

$$
R(x)=\frac{\Delta z\left(1+\mu_{\text {eff }} \rho_{1}\right) \exp \left(-\mu_{\text {eff }} \rho_{1}\right)}{4 \pi \rho_{1}{ }^{3}}+\frac{\left(\Delta z+2 z_{b}\right)\left(1+\mu_{\text {eff }} \rho_{2}\right) \exp \left(-\mu_{\text {eff }} \rho_{2}\right)}{4 \pi \rho_{2}^{3}} \text {. }
$$

where $\rho_{1}$ and $\rho_{2}$ are the distances from the two point sources to the point of interest and

$$
\Delta \mathrm{z}=\frac{\cos \left(\alpha_{\mathrm{s}}\right)}{0.35 \mu_{\mathrm{a}}+\mu_{\mathrm{s}}{ }^{\prime}}=\Delta \mathrm{x} \tan ^{-1}\left(\alpha_{\mathrm{t}}\right)
$$

Equation 2 can be scaled to fit a relative reflectance profile.

Therefore, the first step in our procedure was to measure the diffuse reflectance profile with our fiber optic probe. We used white light to do a multi-wavelength measurement. The light collected by each detection fiber was input to the OMA for spectral resolution. Because we determine the spectrum collected by each fiber, we have in fact measured the spatial distribution of diffuse reflectance at many wavelengths simultaneously. Thus the reflectance profile at any wavelength can be extracted and analyzed to deduce the optical properties at that wavelength.

Once the position of the center of diffuse reflectance relative to the light entry point $(\Delta x)$ was found, the diffusion coefficient was calculated from

$$
D=\frac{\Delta x}{3 \sin \left(\alpha_{t}\right)}
$$


Then a non-linear least squares fit to equation (2) with the Levenberg-Marquardt method $^{8}$ yielded $\mu_{\text {eff }}$

$$
\mu_{\text {eff }}=\sqrt{\mu_{\mathrm{a}} / \mathrm{D}} \text {. }
$$

and the following equations were used to compute for $\mu_{\mathrm{a}}$ and $\mu_{\mathrm{s}}$.

$$
\mu_{\mathrm{a}}=\frac{\mu_{\mathrm{eff}}^{2} \Delta \mathrm{x}}{3 \sin \left(\alpha_{\mathrm{t}}\right)}
$$

and

$$
\mu_{\mathrm{s}}^{\prime}=\frac{\sin \left(\alpha_{\mathrm{t}}\right)}{\Delta \mathrm{x}}-0.35 \mu_{\mathrm{a}}
$$

\section{Results}

In Figure 5 the average absorption for each angle is plotted against the wavelength. The absorption coefficient was consistently lower at $0^{\circ}$ than that at $90^{\circ}$. Two peaks at approximately 450 and 550 were observed in the absorption spectra, which matched the absorption peaks of hemoglobin.

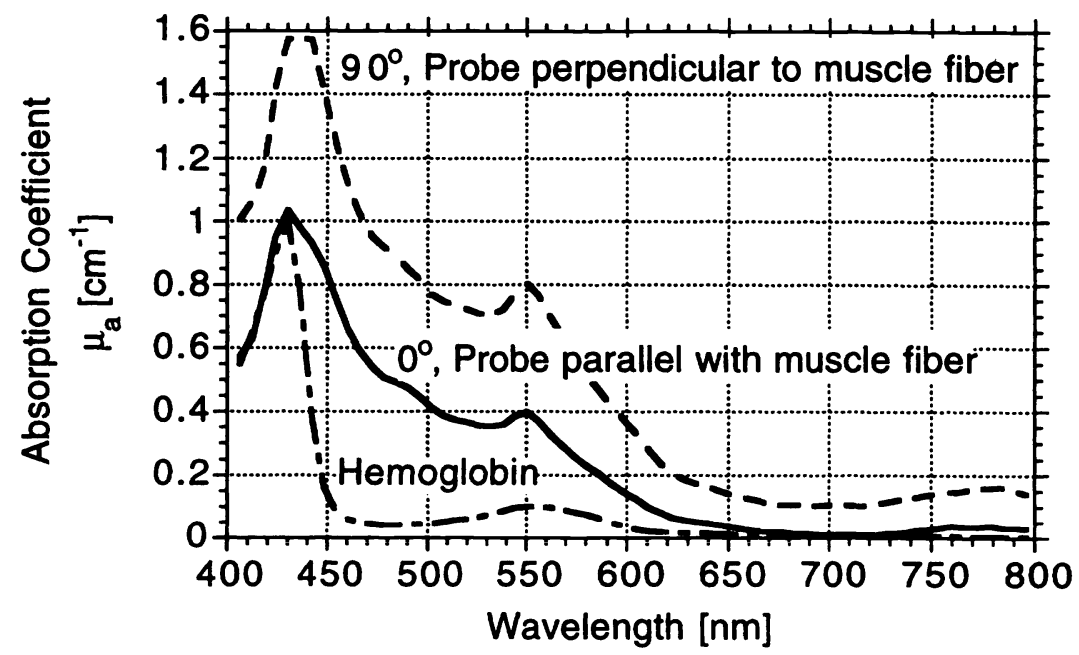

Figure 5. Absorption coefficient vs. wavelength at two orthogonal probe orientations. 
In Figure 6 the average reduced scattering coefficient for each angle is plotted against the wavelength. As a simple comparison, the $90^{\circ}$ curve had a lower $\mu_{\mathrm{s}}^{\prime}$ than the $0^{\circ}$ curve in the low wavelength region. However, there was a crossover between the curves at about $575 \mathrm{~nm}$.

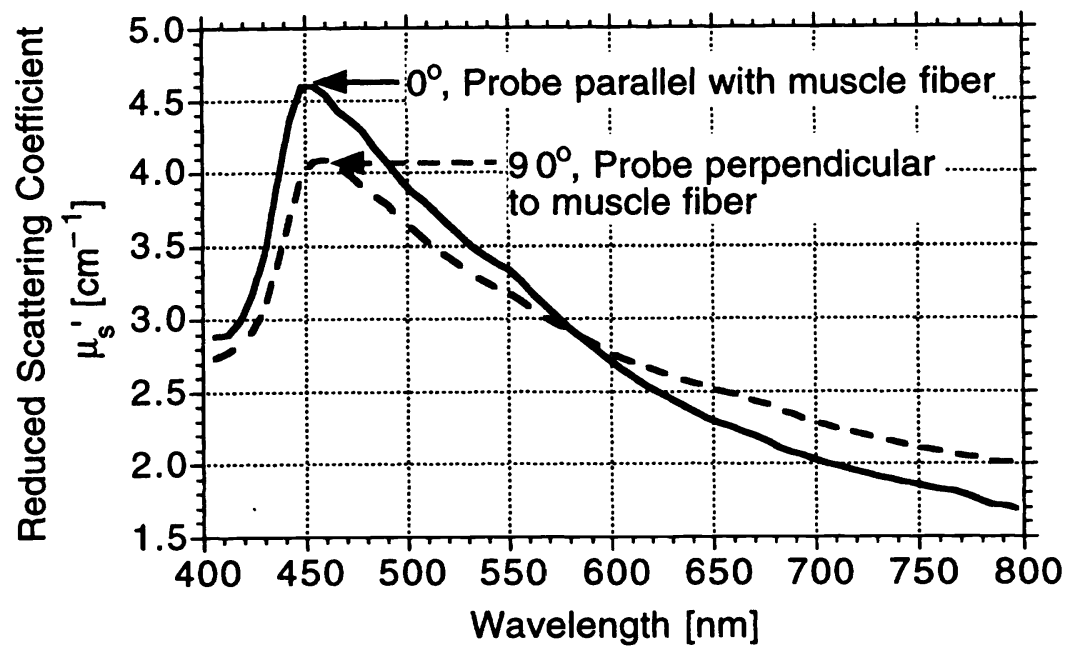

Figure 6. Reduced scattering coefficient vs. wavelength at two orthogonal probe orientations.

To understand what caused the crossing between the two reduced scattering spectra, we computed the reduced scattering spectra due to scattering by spheres of two different sizes (Fig. 7). As can be seen, there was a crossover between the two curves as well. The reduced scattering spectrum due to the small scatterers had a sharper slope than that due to the large scatterers.

\section{Discussion and Conclusions}

As previously stated, oblique incidence reflectometry method can quickly, inexpensively, and noninvasively measure the absorption and reduced scattering coefficients of turbid media. The multi-wavelength measurements were accomplished by extending our monochromatic measurements by using a white light source. In our experiments, we used chicken breast as a prelude to clinical studies. We hope to determine the effect of structured media such as muscle on the optical properties of tissues.

As shown in Figure 5 and Figure 6 we can see differences in the absorption and reduced scattering coefficients due to changes in the angle. The double peaks in the measured absorption 
spectra should be caused by some residual hemoglobin in the chicken breast tissue. The anisotropic absorption property of the muscle tissue was probably caused by the anisotropic arrangement of absorbers such as hemoglobin. How much the absorbers are sampled by light depends on the orientation of the probe. The anisotropic scattering property was caused by the muscle fiber alignment. Multiple levels of fiber structures constitute the muscle, ranging from muscle fibers, myofibrils, and myofilaments. The latter two levels have diameters comparable with the wavelength of light and should be the primary contributors to scattering. When the probe was oriented parallel to the muscle fibers, light interacted frequently with the fibers along the fibers and hence crossed small cross sections, i.e., "small scatterers", and vice versa. Because small scatterers cause a sharper slope in reduced scattering spectra (Figure 7), the slope in the reduced scattering spectrum at $0^{\circ}$ was sharper than that at $90^{\circ}$. Time-resolved light transmission through tubular tissues was shown to have a significant difference depending on the relative orientation of the incident light and the tissue structure. ${ }^{9}$

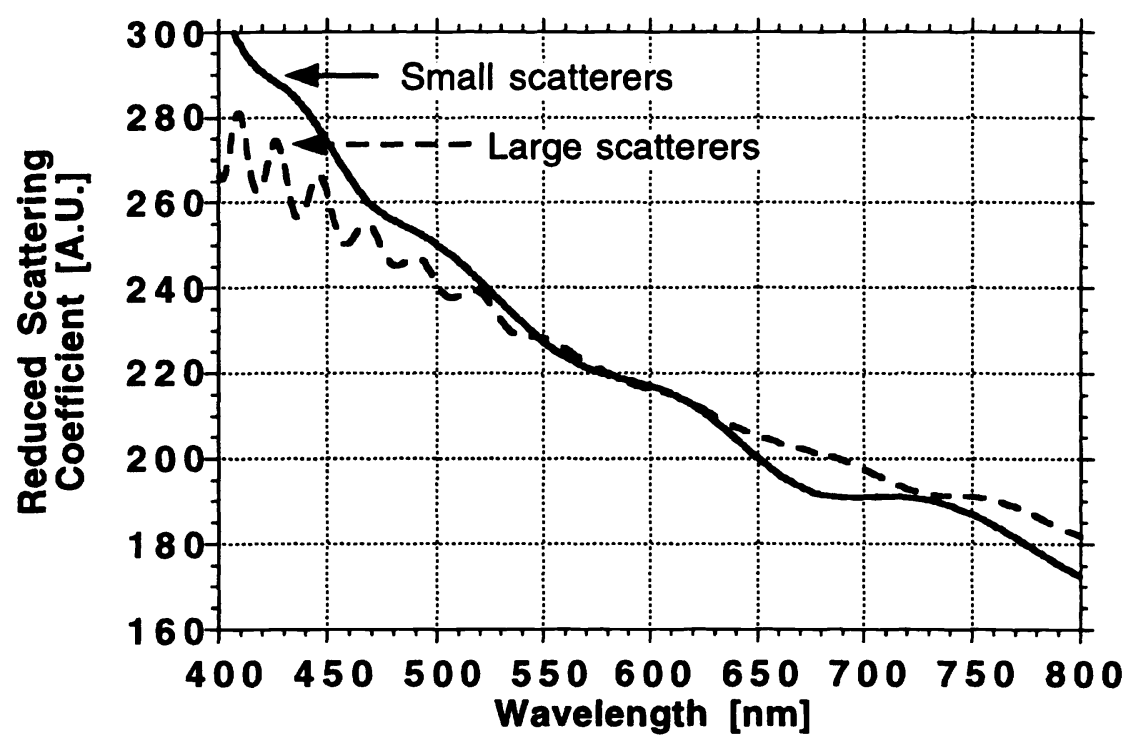

Figure 7. Reduced scattering spectra due to scatterers of two different sizes calculated by Mie theory.

In conclusion this research demonstrated 1) an application of oblique-incidence fiber-optic reflectometry to measurements on biological tissue and 2) the effect of structural anisotropy on optical properties. Our theory was based on the application of diffusion theory to the radiation transport equation. The theory that the propagation of light in the media must be an isotropic point source. However, chicken was not an isotropic media and therefore our absorption measurement 
was rather qualitative than quantitative. The measured reduced scattering coefficient was considered accurate since Equation 7 was not based on $\mu_{\text {eff }}$ and $\mu_{\mathrm{a}}$ was much less than $\mu_{\mathrm{s}}^{\prime}$.

\section{Acknowledgments}

The project was sponsored in part by The Whitaker Foundation grant and the National Institutes of Health grant R29 CA68562 and R01 CA71980. 


\section{Glossary}

\begin{tabular}{|c|c|}
\hline $\begin{array}{l}\text { Absorption coefficient } \\
{\left[\mu_{\mathrm{a}}, \mathrm{cm}^{-1}\right]}\end{array}$ & The probability of photon absorption per unit infinitesimal pathlength. \\
\hline Anisotropy $[\mathrm{g}]$ & $\begin{array}{l}\text { The average of the cosine value of the deflection angle by single } \\
\text { scattering. }\end{array}$ \\
\hline $\begin{array}{l}\text { Diffusion constant } \\
{[\mathrm{D}, \mathrm{cm}]}\end{array}$ & $\begin{array}{l}\text { Linking the gradient of light fluence, } \nabla \phi \text {, and light current, F, (Fick's } \\
\text { law), i.e., } F=-D \nabla \phi \text {. }\end{array}$ \\
\hline $\begin{array}{l}\text { Effective attenuation } \\
\text { coefficient }\left[\mu_{\text {eff }}, \mathrm{cm}^{-1}\right]\end{array}$ & $\begin{array}{l}\text { The decay constant of light fluence far away from light source. } \\
\mu_{\text {eff }}=\sqrt{3 \mu_{\mathrm{a}} / \mathrm{D}} \text {. }\end{array}$ \\
\hline $\begin{array}{l}\text { Interaction coefficient } \\
{\left[\mu_{\mathrm{t}}, \mathrm{cm}^{-1}\right]}\end{array}$ & $\begin{array}{l}\text { The probability of photon interaction per unit infinitesimal pathlength, } \\
\text { where the interaction includes both absorption and scattering. } \mu_{t}=\mu_{a} \\
+\mu_{s} \text {. Sometimes, it is also called total interaction coefficient or total } \\
\text { attenuation coefficient. }\end{array}$ \\
\hline Mean free path [mfp] & The mean pathlength between interactions, which is $1 / \mu_{\mathrm{r}}$. \\
\hline $\begin{array}{l}\text { Penetration depth }[\delta \text {, } \\
\mathrm{cm}]\end{array}$ & $\begin{array}{l}\delta=1 / \mu_{\text {eff }} \text { It represents decay constant of the light fluence far from the } \\
\text { source. }\end{array}$ \\
\hline $\begin{array}{l}\text { Reduced scattering } \\
\text { coefficient }\left[\mu_{\mathrm{s}}^{\prime}, \mathrm{cm}^{-1}\right]\end{array}$ & $\begin{array}{l}\mu_{\mathrm{s}}^{\prime}=\mu_{\mathrm{s}}(1-\mathrm{g}) . \text { Sometimes, it is also called transport scattering } \\
\text { coefficient. }\end{array}$ \\
\hline $\begin{array}{l}\text { Scattering coefficient } \\
{\left[\mu_{\mathrm{s}}, \mathrm{cm}^{-1}\right]}\end{array}$ & The probability of photon scattering per unit infinitesimal pathlength. \\
\hline $\begin{array}{l}\text { Transport interaction } \\
\text { coefficient }\left[\mu_{\imath}^{\prime}, \mathrm{cm}^{-1}\right]\end{array}$ & $\mu_{\mathrm{t}}^{\prime}=\mu_{\mathrm{a}}+\mu_{\mathrm{s}}^{\prime}$ \\
\hline $\begin{array}{l}\text { Transport mean free } \\
\text { path [mfp'] }\end{array}$ & $1 / \mu_{t}^{\prime}$ \\
\hline
\end{tabular}




\section{References}

1. L.-H. Wang and S. L. Jacques, "Use of a laser beam with an oblique angle of incidence to measure the reduced scattering coefficient of a turbid medium," Appl. Opt. 34, 2362-2366 (1995).

2. S.-P. Lin, L.-H. Wang, S. L. Jacques and F. K. Tittle, "Measurement of absorption and scattering spectra with oblique incidence reflectometry," in Advances in Optical Imaging and Photon Migration, OSA Technical Digest Series (Optical Society of America, Washington, D. C., 1996).

3. G. Marquez, L.-H. Wang, S.-P. Lin, S. L. Jacques, F. K. Tittel, S. L. Thomsen, and J. A. Schwartz, "Measurement of absorption and scattering spectra of chicken breast with oblique incidence reflectometry," in Biomedical Sensing, Imaging, and Tracking Technologies II, T. Vo-Dinh, R. A. Lieberman, and G. G. Vurek, eds., Proc. Soc. PhotoOpt. Instrum. Eng. 2976, 306-317 (SPIE Press, 1997).

4. G. Marquez, L.-H. Wang, S.-P. Lin, J. A. Schwartz, and S. L. Thomsen, "Anisotropy in the absorption and scattering spectra of chicken breast tissue," Appl. Opt. 37, 798-805 (1998).

5. J. W. Pickering, S. A. Prahl, N. Vanwieringen, J. F. Beek, H. J. C. M. Sterenborg, and M. J. C. van Gemert, "Double-integrating-sphere system for measuring the optical properties of tissue," Appl. Opt. 32, 399-410 (1993).

6. S. L. Jacques, A. Gutsche, J. A. Schwartz, L.-H. Wang, and F. K. Tittel, "Video reflectometry to specify optical properties of tissue in vivo," in Medical Optical Tomography: Functional Imaging and Monitoring, IS11 of SPIE Institute Series (Soc. Photo-Opt. Instrum. Eng., Bellingham, Wash), 211-226 (1993).

7. T. J. Farrell, M. S. Patterson, and B. C. Wilson, "A diffusion theory model of spatially resolved, steady-state diffuse reflectance for the non-invasive determination of tissue optical properties in vivo," Med. Phys. 19, 879-888 (1992).

8. W. H. Press, B. P. Flannery, S. A. Teukolsky, and W. T. Veterlin, Numerical Recipes in $C$, 2nd ed. (Cambridge U. Press, Cambridge, UK, 1992), Section 15.5.

9. M. E. Zevallos, F. Liu, and R. R. Alfano, "Time-resolved pulse propagation in tissue tubular structures," in 1997 OSA Technical Digest Series 11, 148-149 (1997). 